\title{
Impact of nuclear data uncertainty on safety calculations for spent nuclear fuel geological disposal
}

\author{
J.J. Herrero ${ }^{1, \text { a }}$, D. Rochman ${ }^{1}$, O. Leray ${ }^{1}$, A. Vasiliev ${ }^{1}$, M. Pecchia ${ }^{1}$, H. Ferroukhi ${ }^{1}$, and S. Caruso ${ }^{2}$ \\ 1 Nuclear Energy and Safety Research Department, Paul Scherrer Institut (PSI), Villigen PSI, Switzerland \\ ${ }^{2}$ National Cooperative for the Disposal of Radioactive Waste (NAGRA), Wettingen, Switzerland
}

\begin{abstract}
In the design of a spent nuclear fuel disposal system, one necessary condition is to show that the configuration remains subcritical at time of emplacement but also during long periods covering up to $1,000,000$ years. In the context of criticality safety applying burn-up credit, k-eff eigenvalue calculations are affected by nuclear data uncertainty mainly in the burnup calculations simulating reactor operation and in the criticality calculation for the disposal canister loaded with the spent fuel assemblies. The impact of nuclear data uncertainty should be included in the k-eff value estimation to enforce safety. Estimations of the uncertainty in the discharge compositions from the CASMO5 burn-up calculation phase are employed in the final MCNP6 criticality computations for the intact canister configuration; in between, SERPENT2 is employed to get the spent fuel composition along the decay periods. In this paper, nuclear data uncertainty was propagated by Monte Carlo sampling in the burn-up, decay and criticality calculation phases and representative values for fuel operated in a Swiss PWR plant will be presented as an estimation of its impact.
\end{abstract}

\section{Introduction}

A summarized result of which spent nuclear fuel assemblies can be loaded into the final disposal canister, fulfilling the regulatory limit from the criticality safety point of view, is usually collected in the form of loading curves.

Together with the regulatory limit, the biases and uncertainties in the calculation methodology employed to generate those curves are also included. It is also common engineering practice to simplify the modelling by means of conservative assumptions which are also added to the bias.

In a previous paper [1], a methodology was introduced with the purpose of allowing a realistic calculation of the fuel assembly isotopic inventory evolution both in time and space, comprising the following steps:

- CASMO5-SIMULATE3 standard cycle calculations support CASMO5 depletion calculations with the state points' values calculated from SIMULATE3 for every irradiation cycle at every axial layer and radial node, in order to reconstruct the burnt fuel assembly composition.

- These compositions are input to the code SERPENT2 to compute the decay of the fuel in a time span of one million years.

- At selected time positions, the effective multiplication factor k-eff of the system formed by the spent fuel inside the flooded disposal canister is computed with MCNP6, considering in the fuel composition a limited set of nuclides.

As mentioned, nuclear data is one source of uncertainty in the computed k-eff values which needs to be accounted for.

a e-mail: jose-javier.herrero@psi.ch
The tools employed to propagate the uncertainties in the different steps of the methodology are all based on the Monte Carlo method and briefly described in the paper.

Results computed for a proposed disposal canister model loaded with high enrichment $\mathrm{UO}_{2}$ fuel are given, showing a first estimation of the expected impact on the derived loading curves.

\section{Methodology}

The computational scheme starts from the reference CASMO5-SIMULATE3 core models which are developed and validated for all Swiss reactors and operated cycles within the CMSYS platform [2]. These models provide realistic estimations of the operating histories, including in-core depletion as well as shutdown cooling between cycles.

This information is recovered by the tool BOHR to allow rerunning CASMO5 [4], now with realistic operating conditions. So that pin-by-pin and axially detailed burnt compositions are obtained.

The burnt compositions are input to SERPENT2 to compute the modified isotopic concentrations in the decay period of interest.

Afterwards, by using a tool named COMPLINK [3], a base MCNP canister model loaded with homogeneous fuel can be processed to import the detailed fuel assembly compositions.

The canister model loaded with burnt fuel is then used to compute k-eff after selected decay periods, so as to assess if the system remains subcritical.

Stochastic sampling was used to compute perturbed results considering the uncertainties in the nuclear data 


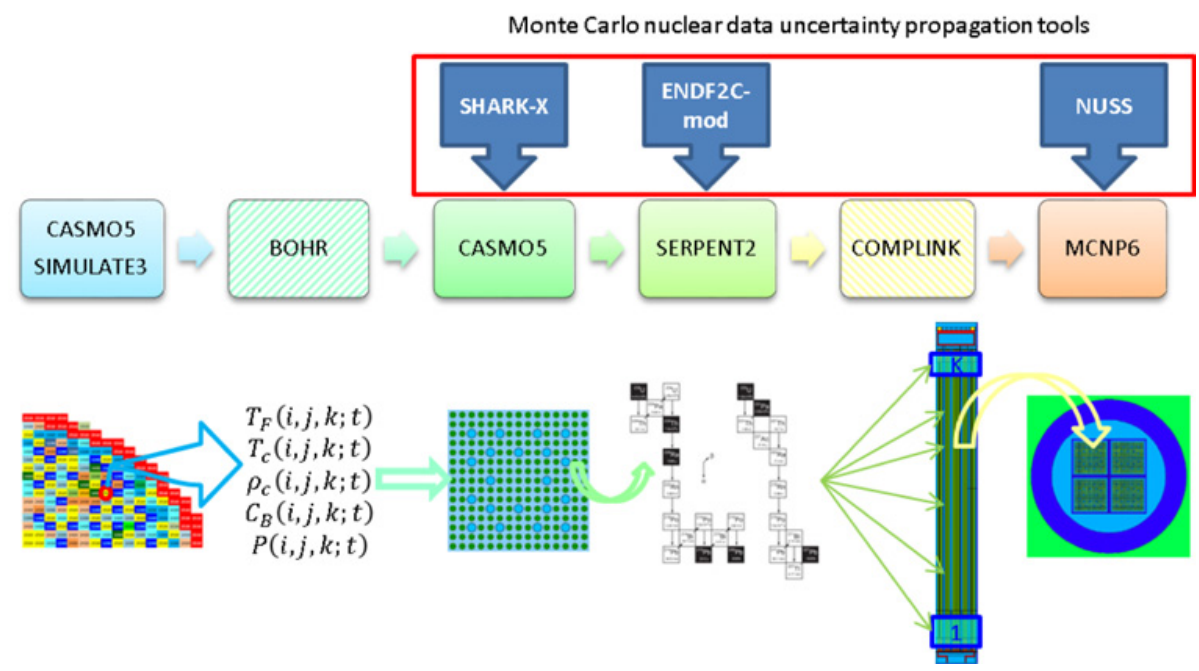

Figure 1. Spent fuel canister k-eff evolution.

by means of separate modules developed for each calculation:

- SHARK-X perturbs nuclear data used in CASMO5.

- ENDF2C perturbs decay data used with SERPENT2.

- NUSS perturbs nuclear data employed in MCNP6.

Figure 1 summarizes the process and next subsections give more details on every step in the methodology.

\subsection{Retrieval of nodal history}

The generation of the data library used by SIMULATE3 is performed with CASMO5 2D lattice depletion calculations for the case matrix of possible operating conditions, which the fuel assembly sectors (nodes) can experience during reactor operation/fuel burnup (temperatures and densities mainly). The nuclear data library employed at this step is based on ENDF/B-VII.0.

The fuel burn-up along the cycle operation is computed with SIMULATE3 using the previous library. From these results, the values of the state parameters are retrieved for every fuel assembly and axial elevation at different cycle instants with a general purpose tool named BOHR. It provides the values of nodal power, fuel temperature, coolant temperature and density, and boron concentration for every location in the nodal geometry. Also the presence of inserted control rods during the operation is taken into account.

\subsection{Lattice calculations for discharge compositions estimation}

In order to get the composition for every fuel pin at the discharge burn-ups for every elevation, new 2D lattice calculations are performed with CASMO5, but now the temperatures and densities employed are the values computed during the SIMULATE3 cycle calculations, and the shutdown cooling periods between cycles are also included. The nuclear data library used is based on ENDF/B-VII.1.

It must be underlined that the CASMO5 reconstructed depletion calculations are performed using single assembly reflected models, i.e., without taking account of a realistic leakage term representative of the $3 \mathrm{D}$ environment under which the assembly was irradiated.

At this stage the module SHARK-X [5] is employed to generate, by stochastic sampling, perturbed values for the nuclear data used by CASMO5. The code uses the covariance matrices from ENDF/B-VII.1 to modify the nuclear data.

Among these data, resonance integrals cannot be perturbed at this point so their impact is lacking; and fission yields are perturbed respecting their physical relationships thus avoiding past misestimations of the uncertainty [5]; finally also decay constants used in the fuel evolution equations are perturbed.

For this study, 170 separate sets of discharge compositions for each irradiation cycle were obtained.

\subsection{Decay calculations after discharge}

From the detailed burn-up results, the fuel compositions change by decay is computed using the Transmutation Trajectory Analysis algorithm programmed in SERPENT2 [6]. The decay data from ENDF/B-VII.1 [7] is employed.

The routine ENDF2C was modified introducing a stochastic sampling perturbation of the decay constants and the branching ratios, whose uncertainties are contained and read by ENDF2C from the original decay data file; and then writing the perturbed decay data files to be employed in SERPENT2. Physical relationship of the branching ratios was respected.

Test calculations using 500 perturbed data files showed a negligible impact on $\mathrm{k}$-eff results $(15 \mathrm{pcm}$ standard deviation) which is in agreement with other published results [8].

\subsection{Criticality calculations for the disposal canister}

The tool COMPLINK [3] developed at PSI is used to generate the model including the spent fuel compositions from a base input file of a canister model loaded with fuel assemblies of equal uniform fresh composition.

Two sets of isotopes are usually considered for burn-up credit calculations, named the actinides only (AC) and the 


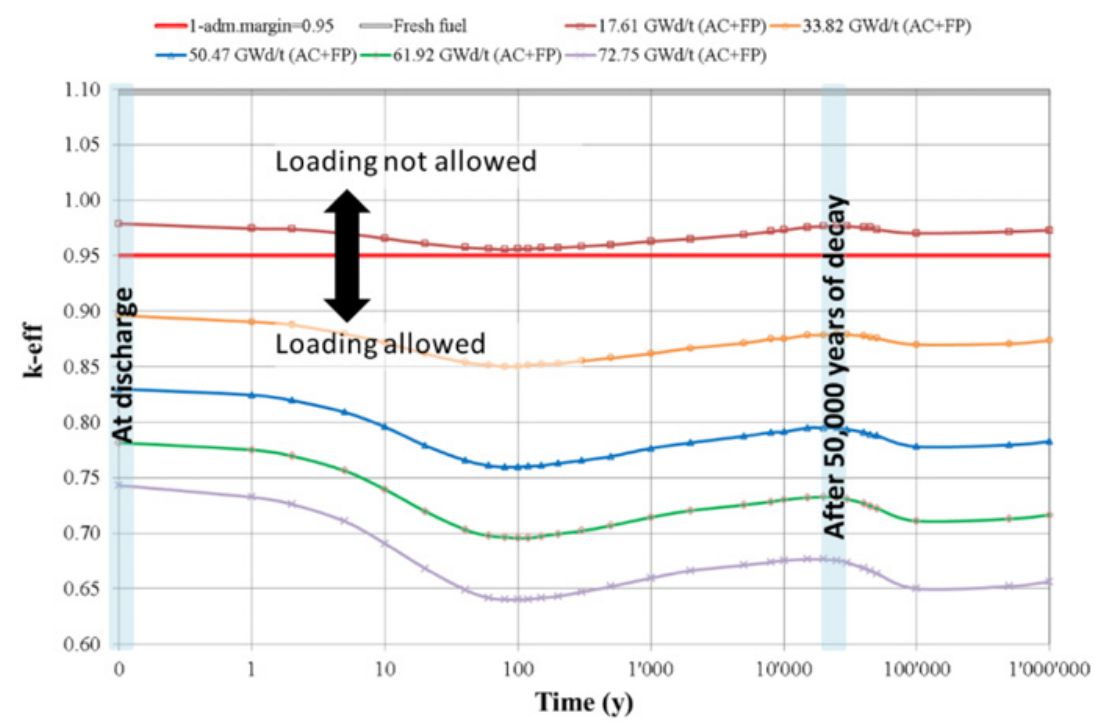

Figure 2. Spent fuel canister k-eff evolution (the limit k-eff $=0.95$ is included just for illustration of safety limit).

Table 1. Set of isotopes included in the criticality calculation.

\begin{tabular}{|c|c|c|c|}
\hline U-233 & U-234 & U-235 & U-236 \\
\hline U-238 & Pu-238 & Pu-239 & Pu-240 \\
\hline Pu-241 & Pu-242 & Am-241 & Cm-243 \\
\hline Cm-244 & Cm-245 & Np-237 & Am-242 \\
\hline Am-243 & Cs-133 & Nd-143 & Nd-145 \\
\hline Sm-147 & Sm-149 & Sm-150 & Sm-151 \\
\hline Sm-152 & Eu-151 & Eu-153 & Gd-155 \\
\hline Mo-95 & Tc-99 & Ru-101 & Rh-103 \\
\hline Ag-109 & & & \\
\hline
\end{tabular}

actinides plus fission products $(\mathrm{AC}+\mathrm{FP})$; Table 1 lists the $\mathrm{AC}+\mathrm{FP}$ set used in the calculations of the results, to which oxygen isotopes $\mathrm{O}-16$ and $\mathrm{O}-17$ are also added.

The nuclear data from ENDF/B-VII.1 coming with the MCNP6 [9] distribution has been used in the calculations. The canister and fuel geometry is considered intact, but in optimal moderation condition (flooded with water). The material temperature is $293.16 \mathrm{~K}$ everywhere and corresponding densities and dimensions are employed.

The neutron multiplication factor, k-eff, is computed with 25 inactive cycles and 30 active cycles to obtain an uncertainty in the range of approximately $\pm 40 \mathrm{pcm}$. All the cycles are run with 200,000 histories each. The number of required inactive cycles was reduced by reusing the source file from the previous calculation.

At this step, there are two sources of uncertainty, one is the nuclear data itself contained in the ACE format files and perturbed by means of the NUSS tool [10], capable of introducing perturbations to (n,n), (n, n'), (n,2n), (n,f), (n, $\gamma)$ cross sections and $(v),(\chi)$ data in the ACE-formatted data files by stochastic sampling of their covariance matrices. 200 different results were computed with perturbed ACE files to estimate the uncertainty on k-eff.

The other source of uncertainty, related to nuclear data, are the 170 different fuel composition sets computed previously with CASMO5 and decayed with SERPENT2, the calculations were performed with the nominal nuclear data ACE file.
The two effects were added together as independent standard deviations. This implies an approximation as the perturbed set of nuclear data used for every discharge composition input to MCNP6 should be consistent with the one employed by CASMO5, some correlation impact is lost in this way.

\section{Results}

The canister is basically a carbon steel cylinder, almost 5 meters height ready to fit 4 PWR fuel assemblies in separate carbon steel boxes inserted and welded [11], no strong neutron absorbers are included in the design. As a worst scenario, without considering degradation issues or damages that could occur during the considered geological periods, the loaded canister is assumed to be flooded with water entering through a postulated breach. Additionally, a $35 \mathrm{~cm}$ layer of water saturated bentonite clay is also included in the model; although its impact on the results will be negligible for the flooded conditions problem.

The 4 assemblies were assumed to have the same nuclear and mechanical design, selected to correspond to the highest enrichment of all assemblies that have been operated up to five cycles (i.e., reaching high burn-ups) at the given plant. For one representative assembly of the given batch, the irradiation history was then reconstructed based on real plant operational data.

The fuel assembly is formed by a $15 \times 15$ array of fuel pins which contain UO2 fuel enriched at $4.94 \mathrm{w} / 0$ and operated during 5 cycles, reaching discharge burn-ups of $17.61,33.82,50.47,61.92$ and $72.75 \mathrm{GWd} / \mathrm{tHM}$. There are 20 guide tubes in the array.

Analyses were conducted for each of the mentioned assembly averaged discharge burn-ups. The curves are plotted in Fig. 2 for the reactivity at different moments after discharge. The observed behavior is in line with classical published results for the same situation [12].

Along the decay period there are two regions where the criticality of the system reaches maxima, just after discharge and after around 50,000 years of decay. The maximum of both is taken for every discharge burn-up and 
Table 2. Nominal and bounding k-eff from nuclear data uncertainty impact just after discharge.

\begin{tabular}{|l|c|c|c|c|}
\hline $\begin{array}{l}\text { Exposure } \\
(\mathbf{G W d} / \mathbf{t H M})\end{array}$ & k-eff & $\begin{array}{c}\text { Isotopic } \\
\text { unc }(\mathbf{2} \boldsymbol{\sigma})\end{array}$ & $\begin{array}{c}\text { ND unc } \\
\mathbf{( 2} \boldsymbol{\sigma})\end{array}$ & $\begin{array}{c}\text { Bounding } \\
\text { k-eff }\end{array}$ \\
\hline 0 & 1.09517 & 0.00000 & 0.00734 & 1.10251 \\
\hline 17.61 & 0.98254 & 0.00324 & 0.00622 & 0.98955 \\
\hline 33.82 & 0.90179 & 0.00516 & 0.00608 & 0.90976 \\
\hline 50.47 & 0.83507 & 0.00716 & 0.00596 & 0.84439 \\
\hline 61.92 & 0.78490 & 0.00874 & 0.00590 & 0.79545 \\
\hline 72.75 & 0.74209 & 0.01052 & 0.00576 & 0.75408 \\
\hline
\end{tabular}

Table 3. Nominal and bounding k-eff from nuclear data uncertainty impact after 50,000 years decay.

\begin{tabular}{|l|c|c|c|c|}
\hline $\begin{array}{l}\text { Exposure } \\
(\mathbf{G W d} / \mathbf{t H M})\end{array}$ & k-eff & $\begin{array}{c}\text { Isotopic } \\
\text { unc }(\mathbf{2} \boldsymbol{\sigma})\end{array}$ & $\begin{array}{c}\text { ND unc } \\
(\mathbf{2} \boldsymbol{\sigma})\end{array}$ & $\begin{array}{c}\text { Bounding } \\
\text { k-eff }\end{array}$ \\
\hline 0 & 1.09517 & 0.00000 & 0.00734 & 1.10251 \\
\hline 17.61 & 0.97335 & 0.00498 & 0.00664 & 0.98165 \\
\hline 33.82 & 0.87758 & 0.00792 & 0.00636 & 0.88774 \\
\hline 50.47 & 0.78677 & 0.01072 & 0.00602 & 0.79906 \\
\hline 61.92 & 0.72220 & 0.01270 & 0.00574 & 0.73614 \\
\hline 72.75 & 0.66346 & 0.01476 & 0.00542 & 0.67918 \\
\hline
\end{tabular}

its dependence fitted to a quadratic function. The values used at fuel discharge are listed in Table 2.

Table 3 shows the k-eff values for the maxima after 50,000 years of decay.

The direct effect from nuclear data in the MCNP6 calculation is similar in both periods and slightly decreasing with the burn-up level attained, and the indirect effect of nuclear data contained in the isotopic uncertainties slightly increases with decay time for a given burnup. These observations are valid for $\mathrm{UO}_{2}$ fuel.

\section{Conclusions}

The calculation route available at PSI to compute k-eff values for spent fuel canisters has been complemented with Monte Carlo uncertainty propagation tools for every step.

The methodology was applied to a canister design being considered in Switzerland for geological disposal, using a high enrichment fuel assembly operated in a Swiss PWR plant.

The results showed that for a burnup credit application on criticality safety, the impact of nuclear data uncertainty through the spent fuel composition, considered as $2 \sigma$ standard deviation, could amount for as much as $1500 \mathrm{pcm}$; and directly in the criticality calculation for as much as $750 \mathrm{pcm}$.

This work has been partially funded by NAGRA, the Swiss organization for waste management.

\section{References}

[1] J.J. Herrero et al., "Computational Scheme for Burnup Credit Applied to Long Term Waste Disposal" in ICNC (2015)

[2] H. Ferroukhi et al., "Core Modelling and Analysis of the Swiss Nuclear Power Plants for Qualified R\&D Applications" in PHYSOR (2008)

[3] M. Pecchia et al., "COMPLINK: A Versatile Tool for Automatizing the Representation of Fuel Compositions in MCNP Models" in ICNC (2015)

[4] "CASMO5. A fuel assembly burn-up program. Methodology Manual", Studsvik, 08/405 Rev 1 (2011)

[5] O. Leray et al., Ann. Nucl. Energy 94, 603 (2016)

[6] J. Leppänen, "Serpent - a Continuous-energy Monte Carlo Reactor Physics Burn-up Calculation Code. User's Manual", VTT (2013)

[7] M.B.B. Chadwick et al., Nucl. Data Sheets 112(12), 2887 (2011)

[8] C.J. Diez et al., Ann. Nucl. Energy 77, 101 (2015)

[9] D.B. Pelowitz et al., "MCNP6 USER'S MANUAL. Code Version 6.1.1beta", Los Alamos, 14-00745 (2014)

[10] T. Zhu et al., Ann. Nucl. Energy 75, 713 (2015)

[11] R. Patel, C. Punshon, J. Nicholas, "Canister Design Concepts for Disposal of Spent Fuel and High Level Waste", NAGRA, NTB 12-06 (2012)

[12] J.C. Wagner, C.V. Parks, "Recommendations on the Credit for Cooling Time in PWR Burn-up Credit Analyses", US-NRC, CR-6781 (2003) 